\title{
Are the Nuclei of Seyfert 2 Galaxies Viewed Face-On?
}

\author{
K. A. Weaver \\ Johns Hopkins University, Department of Physics and Astronomy, Homewood Campus, \\ 3400 North Charles Street, Baltimore, MD 21218-2695; kweaver@pha.jhu.edu \\ and \\ C. S. Reynolds \\ JILA, Campus Box 440, University of Colorado, Boulder, CO 80309-0440
}

Received __; accepted _ _ 


\begin{abstract}
We show from modeling the $\mathrm{Fe} \mathrm{K} \alpha$ line in the $A S C A$ spectra of four X-ray bright narrow emission line galaxies (Seyfert types 1.9 and 2) that two equally viable physical models can describe the observed line profile. The first is discussed by Turner et al. (1998) and consists of emission from a nearly pole-on accretion disk. The second, which is statistically preferred, is a superposition of emission from an accretion disk viewed at an intermediate inclination of $\sim 48^{\circ}$ and a distinct, unresolved feature that presumably originates some distance from the galaxy nucleus. The intermediate inclination is entirely consistent with unified schemes and our findings challenge recent assertions that Seyfert 2 galaxies are preferentially viewed with their inner regions face-on. We derive mean equivalent widths for the narrow and disk lines of $<\mathrm{EQW}_{\mathrm{N}}>=60 \mathrm{eV}$ and $<\mathrm{EQW}_{\mathrm{D}}>=213 \mathrm{eV}$, respectively. The X-ray data are well described by a geometry in which our view of the active nucleus intersects and is blocked by the outer edges of the obscuring torus, and therefore do not require severe misalignments between the accretion disk and the torus.
\end{abstract}

Subject headings: galaxies: active - galaxies: nuclei - galaxies: Seyfert - X-rays: galaxies 


\section{Introduction}

For over a decade, the paradigm for active galactic nuclei (AGN) has rested soundly on the unified model hypothesis, which posits that the only difference between broad-line objects (e.g., Type 1 Seyfert galaxies) and narrow-line objects (e.g., Type 2 Seyferts) is that in the former case our line of sight evades obscuration by the optically thick torus surrounding the nucleus, while in the latter, our line of sight intersects and is blocked by the torus. Assuming that the torus and inner accretion disk are co-planar, this hypothesis makes testable predictions about the shape of the Fe $\mathrm{K} \alpha$ emission line profile, which is commonly very strong in Seyfert galaxies and can yield important information about the orientation of the system relative to our line of sight, as well as the geometry and dynamics of the emitting matter. In the standard picture of Seyfert galaxies, when looking along or near the axis for a face-on system, we expect the $\mathrm{K} \alpha$ photons to arise mostly in an accretion disk resulting in a line profile that is broadened by gravitational and Doppler effects (e.g., George \& Fabian 1991; Laor 1991). When looking in the equatorial plane, the prediction of the standard model for the Fe K $\alpha$ line depends on the optical depth and geometry of the obscuring matter. If the column density is small enough to let X-rays of energy greater than a few keV go through, we would see a very broad, but relatively weak contribution from the disk, and a relatively strong, narrow component from the obscuring torus (Ghisellini, Haardt \& Matt 1994; hereafter GHM94, Krolik, Madau \& Zycki 1994; hereafter KMZ94). In all cases, a weak component from fluorescence in the clouds making up the broad-line region may also be present (Leahy \& Creighton 1993).

The standard unified model has recently been brought into question by studies of the detailed shape of the Fe K $\alpha$ emission line (Turner et al. 1998, hereafter T98). These authors argue that in Type 2 Seyfert galaxies with small enough absorbing columns that the $6 \mathrm{keV}$ nuclear continuum can be seen directly, there are $\mathrm{K} \alpha$ lines with enough breadth 
to suggest that they are made in an accretion disk, but so narrow and asymmetric that the inferred viewing direction is nearly polar and consistent with the mean inclination angle for Seyfert 1s (Nandra et al. 1997). If these studies are borne out, it would mean that in some Type 2 Seyferts a relatively small amount of absorption is present, but lies on the disk axis rather than solely in its equatorial plane, or that the disk axis is severely misaligned with respect to the the galaxy axis and the absorption arises in the galaxy instead of the torus. Furthermore, since Nandra et al. (1997) showed that Seyfert 1 nuclei also have iron line profiles indicative of face-on disks, the T98 hypothesis would force us to consider an entire missing population of AGN containing edge-on disks.

This result has far-reaching consequences for studies of AGN and may force us to re-examine, if not altogether abandon, many of our assumptions for current unified models. However, X-ray spectral data are often subject to multiple interpretations. The conclusion reached by T98 that Type 2 Seyfert galaxies are preferentially viewed with their central regions face-on is based on data from the $A S C A$ satellite (Tanaka, Inoue \& Holt 1994); primarily from spectral modeling of four X-ray selected Type 2 and 1.9 Seyfert galaxies (historically referred to as narrow emission line galaxies or NELGs). In this paper, we seek to determine how strongly these data require the central regions of Seyfert $2 \mathrm{~s}$ to be viewed preferentially face-on by examining a physically plausible modeling of the $\mathrm{Fe} \mathrm{K} \alpha$ line that is consistent with unified schemes. We re-examine the $A S C A$ data and use an alternate modeling technique that allows significant contributions to the Fe K $\alpha$ line from both the accretion disk and the torus.

\section{Data analysis and Results}

The $A S C A$ data are taken from the public archive. We try as much as possible to reproduce the T98 dataset by selecting the same observations and adopting the same criteria 
for data selection. The AGN in this sample possess flux in excess of the extrapolation of a uniformly absorbed power-law to energies below $2 \mathrm{keV}$ (i.e., a "soft excess", T98). To avoid complexities of modeling the soft excess, we constrain our fits to data between 2 and 10 $\mathrm{keV}$ and use a baseline continuum model that consists of a power law (PL) attenuated by uniform, neutral absorption. We list the value of $\chi^{2}$ and the number of degrees of freedom for the PL fits in Table 1, column 3. We do not quote model parameters for these baseline fits because they are similar to those quoted by Turner et al. (1997) and are unimportant for our study.

The $\mathrm{Fe} \mathrm{K} \alpha$ lines in these galaxies are strong and resolved with $A S C A$, and have been examined in detail by other authors (T98, MCG-5-23-16: Weaver et al. 1997, NGC 7314: Yaqoob et al. 1996, NGC 2110: Hayashi et al. 1996). In Table 1 we show results for fits that include a single, unresolved (narrow) Gaussian at a rest energy of $6.4 \mathrm{keV}$ to represent fluorescence of neutral iron. Including a Gaussian improves all fits at $>>99 \%$ confidence compared to the PL fits (col. 3), thus confirming the significance of the Fe $\mathrm{K} \alpha$ lines. Figure 1 shows the co-added line profile measured with the Solid-State Imaging Spectrometer (SIS) detectors (see also T98, Fig. 2). The profile peaks strongly at the center, which coincides with $6.4 \mathrm{keV}$ (rest energy), and emission on either side of the core from $\sim 4.5$ to $8 \mathrm{keV}$ is clearly present. The line shape is, in fact, very different from some Seyfert 1 galaxies, which show a strong red wing but little emission blueward of the line core. One such example is the well-studied galaxy MCG-6-30-15 (Iwasawa et al. 1996).

We next test the standard accretion disk-line models used by T98 (Fabian et al. 1989). These models assume a Schwarzschild geometry and represent the disk emissivity as a power-law function of disk radius, in this case $r^{-2.5}$. We also fix the outer disk radius, $r_{o}$, at $1000 r_{g}$ and fix the inner disk radius, $r_{i}$, at $6 r_{g}$, where $r_{g}=G M / c^{2}$. The free parameters are the inclination of the disk normal to our line of sight $(\theta)$ and the line normalization. 
For disk-line models, the shape of the line changes dramatically with disk inclination as shown in Figure 2. Results of the fits with the PL + disk-line model are presented in Table 2. The standard Schwarzschild disk-line model yields a better fit compared to a narrow Gaussian for three of the four galaxies. We derive a mean inclination for the accretion disk of $\langle\theta\rangle=38^{\circ}$ and a mean equivalent width for the disk line of $\left\langle\mathrm{EQW}_{\mathrm{D}}\right\rangle=300 \mathrm{eV}$. Our inclination is somewhat larger than T98, who find $\langle\theta\rangle=20^{\circ}$ for these data.

We have carefully examined possible reasons for the discrepancy between our derived inclinations and those of T98. These authors do not make clear whether they include a weak narrow component in the disk-line fits they quote, so we also performed the above fits including a weak, narrow Gaussian $\left(\mathrm{EQW}_{\mathrm{N}}<50 \mathrm{eV}\right)$. For this case, the only way to approximate low inclinations is by blueshifting the narrow component so that it is no longer located at the systemic velocity of the galaxy. We can therefore achieve consistency with T98, but only with a model that differs slightly from the one these authors quote. We believe that the difference between our values of $\theta$ and those of T98 are not due to large, intrinsic uncertainties with a particular model (such that both results actually overlap in some overlooked, statistical sense). Indeed, we argue that the T98 model is incomplete and, based on our careful examinations of the data, possibly non-physical.

We believe that a more physical composite model for the Fe $\mathrm{K} \alpha$ line consists of a disk line and a narrow Gaussian with an unrestricted equivalent width $\left(\mathrm{EQW}_{\mathrm{N}}\right)$. The upper limit on $\mathrm{EQW}_{\mathrm{N}}$ of $50 \mathrm{eV}$ that is assumed by T98 is a reasonable assumption for approximately face-on views if the narrow component arises purely in a spherical distribution of clouds (such as the BLR) as predicted from Monte Carlo simulations (Leahy \& Creighton 1993); however, the narrow component may be stronger if the torus contributes, especially as the view of the central region becomes more edge-on (KMZ94). We therefore choose not to restrict $\mathrm{EQW}_{\mathrm{N}}$ in our fits. Results for our composite model are listed in Table 3. In 
all cases, the fits are better than fits with a single disk-Line (Table 2), with probabilities of $\mathrm{P}=0.01$ to 0.001 of exceeding the F-statistic by chance. We derive $\langle\theta\rangle=48^{\circ}$, $<\mathrm{EQW}_{\mathrm{N}}>=60 \mathrm{eV}$, and $<\mathrm{EQW}_{\mathrm{D}}>=213 \mathrm{eV}$.

In Table 4, we compare the accretion-disk parameters inferred for our disk-plus-torus model to those derived by T98 for a pure disk-line description of the Fe $\mathrm{K} \alpha$ line. We derive systematically larger inclinations for the accretion disk and up to $50 \%$ smaller values for $<\mathrm{EQW}_{\mathrm{D}}>$. Both models are plotted together at full resolution in Figure 3. For emission from a disk viewed at an intermediate inclination, significant flux is predicted blueward of the line core. This signature is clearly present in the data (Figure 1) but is absent for the T98 "pole-on" disk model. The two models are, however, qualitatively similar below $6.4 \mathrm{keV}$. Because of this similarity, X-ray data with the restricted bandpass and moderate energy resolution of $A S C A$ (0.6 to $10 \mathrm{keV}$ and $\geq 130 \mathrm{eV}$ FWHM, respectively) make it difficult, if not impossible to distinguish between the two cases. The $A S C A$ data do not necessarily constrain the shape of the continuum above the line and so detecting a blue wing can be highly sensitive to how the continuum is modeled.

\section{Discussion}

We have shown that the Fe K $\alpha$ emission lines in four X-ray selected Type 1.9 and 2 Seyfert galaxies are well modeled as the superposition of two features, a narrow Gaussian at a rest energy of $6.4 \mathrm{keV}$ and an emission line from an accretion disk viewed at an intermediate inclination angle of $\sim 48^{\circ}$. Statistically, this model describes the observed profiles better than a single, highly-peaked emission line from an accretion disk that is seen approximately face-on (suggested by T98). We argue that current moderate-resolution

\footnotetext{
${ }^{1}$ The energy resolution of the SIS detectors has been degrading steadily with time.
} 
X-ray data for Seyfert galaxies cannot easily deconvolve the multiple Fe K $\alpha$ emission components and so cannot necessarily distinguish between these scenarios without variability information or information from other wavebands that suggests the true origin of the line(s). It must be noted that Yaqoob et al. (1996) have found the iron line profile in NGC 7314 to be variable in exactly the manner expected if there is a narrow component of the line that originates at a large distance from the black hole. However, the comparatively low signal to noise of the time-resolved spectra presented by Yaqoob et al. (1996) prevents a detailed decomposition of the narrow and broad line components.

Our results suggest that an intermediate-inclination broad line contaminated by a narrow component can mimic the predicted shape for emission from an approximately face-on accretion disk. T98 dismissed the two-component model because they found that weak narrow components (EQW from $10-50 \mathrm{eV}$ ) had no effect on their fits. We argue that there is no reason to restrict the strength of a contaminating iron line from the molecular torus to have an equivalent width less than $50 \mathrm{eV}$. First of all, we do not know the inclination of the system a priori. There is in fact independent evidence for an intermediate to edge-on orientation for at least one of the NELGs in our sample (MCG-5-23-16; Weaver, Krolik \& Pier 1998). In the case of NELGs viewed at intermediate angles, we would expect our line of sight to intersect the edges of the torus just outside the half opening angle, which is consistent with the moderate line-of-sight column densities of $\sim 10^{22}$ to $\sim 10^{23}$ $\mathrm{cm}^{-2}$. If these measurements represented the mean column density through the torus, $<N_{\mathrm{H}}(\mathrm{T})>$, then $\mathrm{EQW}_{\mathrm{N}}$ would be a few tens of eV for all inclination angles (GHM94) and would contribute only a small fraction of the total line photons. However, the column density through the torus for other lines of sight could easily be as high as $10^{24-25} \mathrm{~cm}^{-2}$. This would result in a larger $<N_{\mathrm{H}}(\mathrm{T})>$ and EQWs of up to $\sim 100 \mathrm{eV}$ for unblocked views of the continuum source, with the actual value depending on the geometry of the torus (KMZ94; GHM94). We find $<\mathrm{EQW}_{\mathrm{N}}>=60 \mathrm{eV}$, which is well within this limit. 
For the disk-line component of the composite model, we derive $\mathrm{EQW}_{\mathrm{D}}$ 's of $\sim 120 \mathrm{eV}$ to $\sim 250 \mathrm{eV}$. These are as much as 50\% smaller than those inferred for the pure disk-line model (this analysis and T98). If, as we suggest, multiple components do comprise the line, then this partly alleviates a fundamental problem that has been frequently pointed out in the literature: the observed EQWs of $\mathrm{Fe} \mathrm{K} \alpha$ lines in Seyfert galaxies are generally larger than the maximum predicted for an accretion disk (e.g., Reynolds \& Fabian 1997). A neutral disk inclined by $\theta=50^{\circ}$ should produce an Fe $\mathrm{K} \alpha$ line with an EQW of $\sim 120$ to $170 \mathrm{eV}$, depending on the value assumed for the solar abundance of iron (George \& Fabian 1991; Reynolds, Fabian \& Inoue 1995). For our model, three of four disk lines have EQWs larger than expected, but only by 50\%. T98 find all four EQWs to be larger than expected, by $\sim 50 \%$ to as much as $\sim 140 \%$. Equivalent widths of more than $\sim 200 \mathrm{eV}$ can result if the disk is ionized (Matt, Fabian, \& Ross 1993), although an ionized disk predicts Fe K $\alpha$ line energies of $\geq 6.6 \mathrm{keV}$, for which there is little evidence (e.g., Nandra et al. 1997). On the other hand, there may be a contribution to the high-energy side of the Fe $\mathrm{K} \alpha$ line from warm scattering material located above the torus, which would reduce the EQW of the disk line even further.

X-ray satellites like $A X A F$ and Astro - E, with much better resolving power, will be able to deconvolve the line and thus will provide the ultimate test of whether the Fe $\mathrm{K} \alpha$ lines of intermediate type Seyfert galaxies are truly complex with distinct, unresolved cores.

\section{Conclusions}

We demonstrate that the Fe K $\alpha$ emission lines for four X-ray selected Seyfert type 1.9 and 2 galaxies are well described with a model that consists of a superposition of emission from an accretion disk viewed at an intermediate angle of $\sim 48^{\circ}$ and a distinct, unresolved emission feature with an equivalent width of $\sim 60 \mathrm{eV}$ that can arise in the obscuring torus. 
This model is entirely consistent with current unified schemes of Seyfert galaxies and therefore does not require severe misalignments between the accretion disk and the torus.

We thank Julian Krolik for reading this manuscript and providing helpful comments and insight. KAW acknowledges the support of NASA LTSA grant NAG5-3504. CSR acknowledges support from NSF grant AST9529175, and NASA LTSA grant NAG5-6337. 


\section{REFERENCES}

Bevington, B. R. 1969, Data Reduction and Error Analysis for the Physical Sciences (McGraw-Hill)

Fabian, A. C., Rees, M. J., Stellar, L. \& White, N. E., 1989, MNRAS, 238, 729

George, I. M. \& Fabian, A. C., 1991, MNRAS, 249, 352

Ghisellini, G., Haardt, F. \& Matt, G., 1994, MNRAS 267, 743

Hayashi, I., Koyama, K., Awaki, H., Yamauchi, S. \& Ueno, S. 1996, PASJ, 48, 219

Iwasawa, K., et al. 1996, MNRAS, 282, 1038

Krolik, J. H., Madau, P. \& Zycki, P. T., 1994, ApJ 420, L57

Laor, A., 1991, ApJ, 376, 90

Leahy, D. A. \& Creighton, J. 1993, MNRAS, 263, 314

Matt, G., Fabian, A. C., \& Ross, R. R. 1993, MNRAS, 262, 179

Nandra, K., George, I. M., Mushotzky, R. F., Turner, T. J. \& Yaqoob T., 1997, ApJ, 477, 602

Reynolds, C. S. \& Fabian, A. C. 1997, MNRAS, 290, L1

Reynolds, C. S., Fabian, A. C., \& Inoue, H. 1997, MNRAS, 276, 1311

Tanaka, Y., Inoue, H. \& Holt, S. S. 1994, PASJ, 46,37

Turner, T. J., George, I. M., Nandra, K. \& Mushotzky, R. F. 1998a, ApJ, 493, 91 (T98)

Turner, T. J., George, I. M., Nandra, K. \& Mushotzky, R. F. 1997, ApJS, 113, 23

Weaver, K. A., Yaqoob, T., Mushotzky, R. F., Nousek, J., Hayashi, I. \& Koyama, K. 1997, ApJ 474, 675

Weaver, K. A., Krolik, J. H., \& Pier, E. A. 1998, ApJ, 498, 213 
Yaqoob, T., Serlemitsos, P. J., Turner, T. J., George, I. M. \& Nandra, K., 1996, ApJ, 470, L27 
Table 1. ASCA Results for Power-law and Narrow Gaussian Fits ${ }^{1}$

\begin{tabular}{ccccccccc}
\hline \hline Galaxy & $\mathrm{z}$ & $\chi_{\mathrm{PL}}^{2} / \nu^{2}$ & $N_{\mathrm{H}}^{3}$ & $\Gamma^{4}$ & $\mathrm{EQW}_{\mathrm{N}}^{5}$ & $\chi_{\mathrm{G}}^{2} / \nu^{6}$ & $\Delta \chi^{27}$ & $\mathrm{~F}^{8}$ \\
\hline & & & & & & & & \\
NGC 526A & 0.0192 & $1509 / 1512$ & $12.7_{-0.8}^{+0.9}$ & $1.65_{-0.03}^{+0.02}$ & $113_{-25}^{+9}$ & $1453 / 1511$ & 56 & 58.3 \\
NGC 2110 & 0.0076 & $815 / 757$ & $33.9 \pm 1.4$ & $1.46_{-0.04}^{+0.03}$ & $140_{-17}^{+18}$ & $748 / 756$ & 67 & 67.7 \\
MCG-5-23 & 0.0083 & $1435 / 1247$ & $14.1_{-0.7}^{+0.6}$ & $1.73 \pm 0.02$ & $93 \pm 10$ & $1349 / 1246$ & 86 & 79.6 \\
NGC 7314 & 0.0047 & $1581 / 1461$ & $5.7_{-0.8}^{+0.6}$ & $1.85 \pm 0.03$ & $120_{-22}^{+12}$ & $1530 / 1460$ & 51 & 48.6 \\
& & & & & & & & \\
\hline
\end{tabular}

${ }^{1}$ The data and selection criteria are identical to those used by T98. Data are fitted from 2 to 10 $\mathrm{keV}$ and errors are 1- $\sigma$. Spectra are grouped to have $>20$ counts per bin. The Fe K $\alpha$ line energy is fixed at $6.4 \mathrm{keV}$ in the galaxy rest frame and the line width is fixed at $0.01 \mathrm{keV}$.

${ }^{2}$ Value of the $\chi^{2}$ statistic divided by the number of degrees of freedom for the power-law (PL) fit (no Gaussian).

${ }^{3}$ Absorbing column density for the PL plus narrow Gaussian fit in units of $10^{21} \mathrm{~cm}^{-2}$.

${ }^{4}$ Photon index for the PL plus Gaussian fit.

${ }^{5}$ Equivalent width of the Gaussian in units of $\mathrm{eV}$.

${ }^{6}$ Value of the $\chi^{2}$ statistic divided by the number of degrees of freedom for the PL plus Gaussian fit.

${ }^{7}$ Change in $\chi^{2}$ for PL plus Gaussian fit compared to the PL fit (col. 3).

${ }^{8}$ F-statistic (Bevington 1969). 
Table 2. Results for Disk-line Fits ${ }^{1}$

\begin{tabular}{ccccccccc}
\hline \hline Galaxy & $N_{\mathrm{H}}$ & $\Gamma$ & $\theta^{2}$ & $\mathrm{EQW}_{\mathrm{D}}^{3}$ & $\chi^{2} / \nu^{4}$ & $\Delta \chi^{25}$ & $\mathrm{~F}^{6}$ & Flux $^{7}$ \\
\hline \multirow{2}{*}{ NGC 526A } & $13.2 \pm 0.9$ & $1.69 \pm 0.03$ & $34_{-3}^{+4}$ & $230_{-40}^{+30}$ & $1454 / 1510$ & -1 & $\ldots$ & 3.6 \\
NGC 2110 & $34.3_{-1.6}^{+1.4}$ & $1.51 \pm 0.04$ & $33_{-4}^{+3}$ & $324_{-40}^{+46}$ & $744 / 755$ & 4 & 4.1 & 3.0 \\
MCG-5-23 & $14.9_{-0.6}^{+0.7}$ & $1.79_{-0.03}^{+0.02}$ & $38_{-4}^{+5}$ & $270 \pm 30$ & $1326 / 1245$ & 23 & 21.5 & 8.7 \\
NGC 7314 & $7.3_{-0.9}^{+0.8}$ & $1.95_{-0.04}^{+0.03}$ & $45_{-3}^{+2}$ & $360 \pm 50$ & $1512 / 1459$ & 18 & 17.3 & 3.6 \\
& & & & & & & & \\
\hline
\end{tabular}

${ }^{1}$ Disk emissivity index $q$ is fixed at -2.5 . Errors are $1-\sigma$. The outer and inner disk radii are fixed at $1000 r_{g}$ and $6 r_{g}\left(r_{g}=G M / c^{2}\right)$. Line energies are $6.4 \mathrm{keV}$ in the galaxy rest frame. The units for $N_{\mathrm{H}}$ are $10^{21} \mathrm{~cm}^{-2}$.

${ }^{2}$ Inclination of the disk normal to our line of sight in units of degrees $\left(\theta=0^{\circ}\right.$ is face-on).

${ }^{3}$ The equivalent width of the disk line in units of $\mathrm{eV}$.

${ }^{4}$ Value of the $\chi^{2}$ statistic divided by the number of degrees of freedom.

${ }^{5}$ Change in $\chi^{2}$ for this fit compared to the narrow Gaussian fit (Table 1).

${ }^{6}$ F-statistic.

${ }^{7}$ The 2 to $10 \mathrm{keV}$ observed flux in units of $10^{-11} \mathrm{ergs}^{-2} \mathrm{~s}^{-1}$. 
Table 3. Results for Disk-Line Plus Narrow Gaussian Fits ${ }^{1}$

\begin{tabular}{ccccccccc}
\hline \hline Galaxy & & & & & & & & \\
$\mathrm{H}$ & $\Gamma$ & $\theta^{2}$ & $\mathrm{EQW}_{\mathrm{D}}^{3}$ & $\mathrm{EQW}_{\mathrm{N}}^{3}$ & $\chi^{2} / \nu^{4}$ & $\Delta \chi^{25}$ & $\mathrm{~F}^{6}$ \\
\hline NGC 526A & $13.3 \pm 0.9$ & $1.68_{-0.02}^{+0.03}$ & $42_{-6}^{+13}$ & $120 \pm 40$ & $64_{-19}^{+17}$ & $1444 / 1509$ & 10 & 10.4 \\
NGC 2110 & $35.3_{-1.6}^{+1.4}$ & $1.54_{-0.04}^{+0.05}$ & $50_{-4}^{+3}$ & $246_{-28}^{+57}$ & $81_{-21}^{+16}$ & $728 / 754$ & 16 & 16.5 \\
MCG -5-23 & $15.5_{-0.7}^{+0.6}$ & $1.81_{-0.03}^{+0.02}$ & $52 \pm 4$ & $235 \pm 35$ & $50_{-11}^{+9}$ & $1309 / 1244$ & 17 & 16.2 \\
NGC 7314 & $7.2_{-1.0}^{+0.7}$ & $1.94_{-0.04}^{+0.02}$ & $46_{-2}^{+5}$ & $250 \pm 50$ & $46_{-15}^{+19}$ & $1505 / 1458$ & 7 & 6.8 \\
& & & & & & & & \\
\hline
\end{tabular}

${ }^{1}$ Errors are $1-\sigma$. The disk emissivity index $q$ is fixed at $-2.5 ; r_{i}=6 r_{g}, r_{o}=1000 r_{g}$. Line energies are $6.4 \mathrm{keV}$ in the galaxy rest frame.

${ }^{2}$ Disk inclination in units of degrees.

${ }^{3}$ Equivalent widths of the disk line and narrow Gaussian $\left(\sigma_{\mathrm{N}}=0.01 \mathrm{keV}\right)$.

${ }^{4}$ Value of the $\chi^{2}$ statistic divided by the number of degrees of freedom.

${ }^{5}$ Change in $\chi^{2}$ compared to the disk-line fits (Table 2).

${ }^{6}$ F-statistic. 
Table 4. Comparing Our Results with T98 $8^{1,2}$

\begin{tabular}{lccccc}
\hline \hline & & & & & \\
\multirow{2}{*}{ This paper } & \multicolumn{2}{c}{ T98 } & \\
& $\theta$ & EQW $_{\mathrm{D}}$ & $\theta$ & $\mathrm{EQW}_{\mathrm{D}}$ & $b / a^{3}$ \\
& $\left(^{\circ}\right)$ & $(\mathrm{eV})$ & $\left(^{\circ}\right)$ & $(\mathrm{eV})$ & \\
\hline & & & & & \\
NGC 526A & $42_{-6}^{+13}$ & $120 \pm 40$ & $13_{-13}^{+6}$ & $281 \pm 56$ & 0.7 \\
NGC 2110 & $50_{-4}^{+3}$ & $246_{-28}^{+57}$ & $15_{-7}^{+9}$ & $289_{-52}^{+59}$ & 0.79 \\
MCG-5-23 & $52 \pm 4$ & $235 \pm 35$ & $33_{-4}^{+11}$ & $362_{-43}^{+94}$ & 0.45 \\
NGC 7314 & $46_{-2}^{+5}$ & $250 \pm 50$ & $17_{-7}^{+6}$ & $417 \pm 82$ & 0.43 \\
$\mu+\sigma$ & $48 \pm 4$ & $213 \pm 62$ & $20 \pm 9$ & $337 \pm 64$ & $\ldots$ \\
& & & & & \\
\hline
\end{tabular}

${ }^{1}$ Line energies are fixed at the rest energy of $6.4 \mathrm{keV}$; the disk emissivity index is $q=-2.5$; the inner and outer disk radii are $6 r_{g}$ and $1000 r_{g}$, respectively $\left(r_{g}=G M / c^{2}\right)$.

${ }^{2}$ The equivalent width for the narrow Gaussian component is a free parameter for our fits and constrained to $<50 \mathrm{eV}$ for the T98 fits. We derive $<\mathrm{EQW}_{\mathrm{N}}>=60 \mathrm{eV}$.

${ }^{3}$ Axial ratio of host galaxy. 


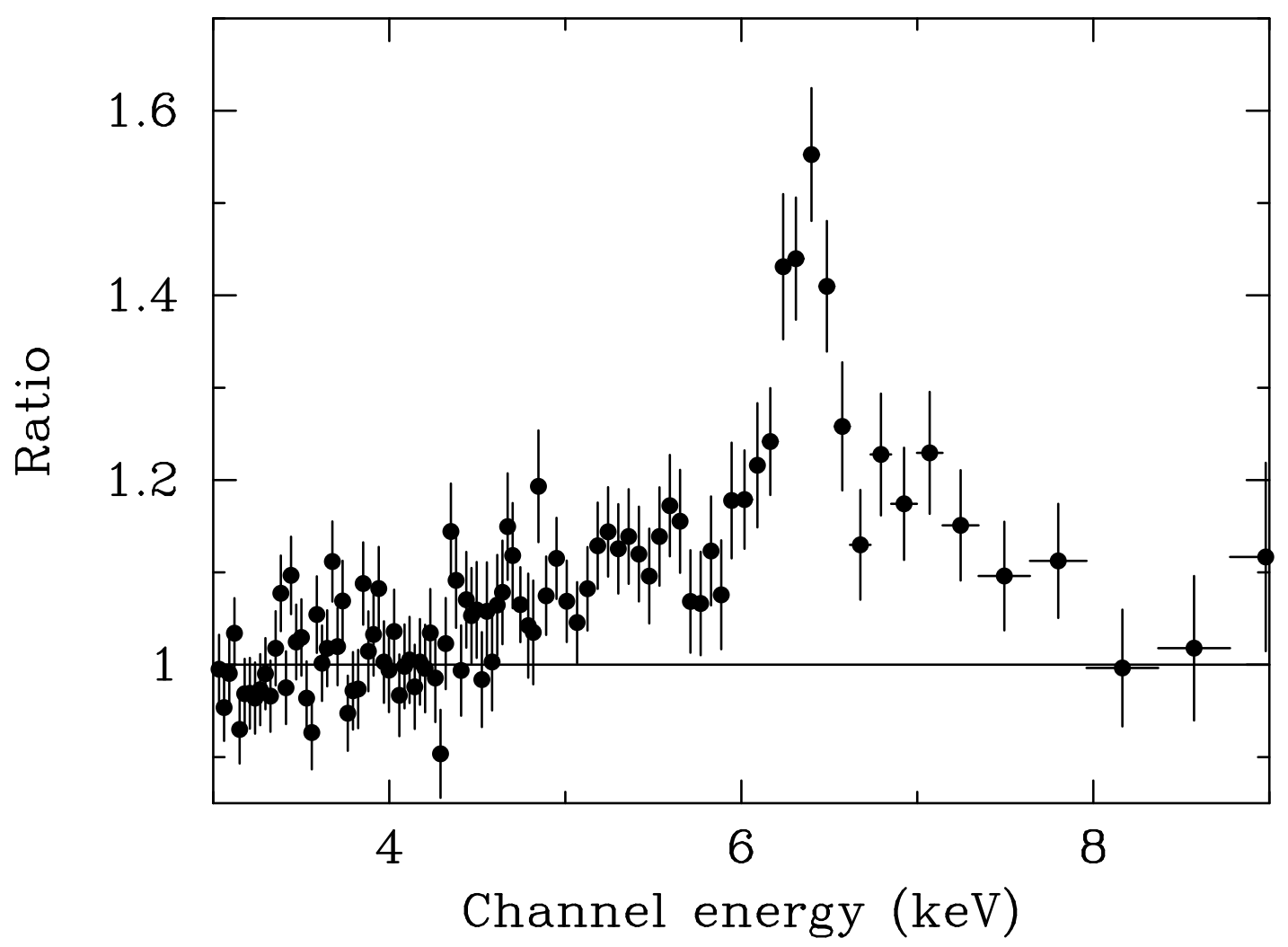

Fig. 1.- Average ratio of the $A S C A$ Solid-State Imaging Spectrometer (SIS) data to the best-fitting continuum models. Individual spectra were corrected for redshift and the data above and below the Fe $\mathrm{K} \alpha$ line (2 to $4.5 \mathrm{keV}$ and 8 to $10 \mathrm{keV}$ ) were fitted with a power law model to estimate the continuum level. The ratios of the data to this model for each galaxy were then calculated and averaged together with xspec (see also T98 Fig. 2, group B). 


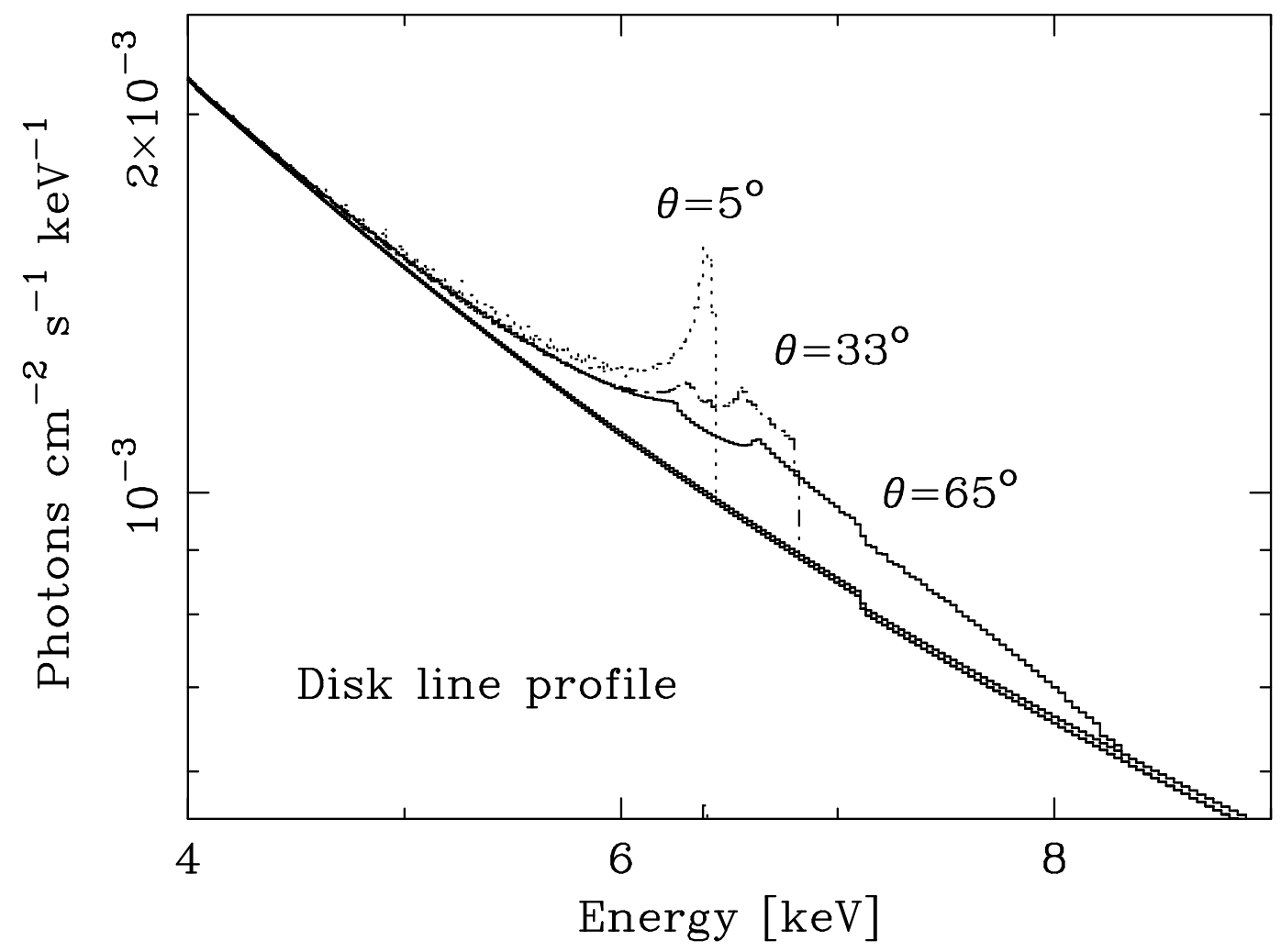

Fig. 2.- Theoretical line profiles from a relativistic accretion disk (Fabian et al. 1989) to illustrate how the profile changes as the inclination of the disk normal to our line of sight increases. Inclinations are $5^{\circ}$ (dotted), $33^{\circ}$ (dashed), and $65^{\circ}$ (solid). Other line parameters are $\mathrm{E}_{\text {peak }}=6.4 \mathrm{keV}$, disk emissivity index, $q=-2.5\left(\mathrm{r}^{q}\right)$, inner radius, $r_{i}=6 r_{g}$, and outer radius, $r_{o}=1000 r_{g}\left(r_{g}=G M / c^{2}\right)$. 


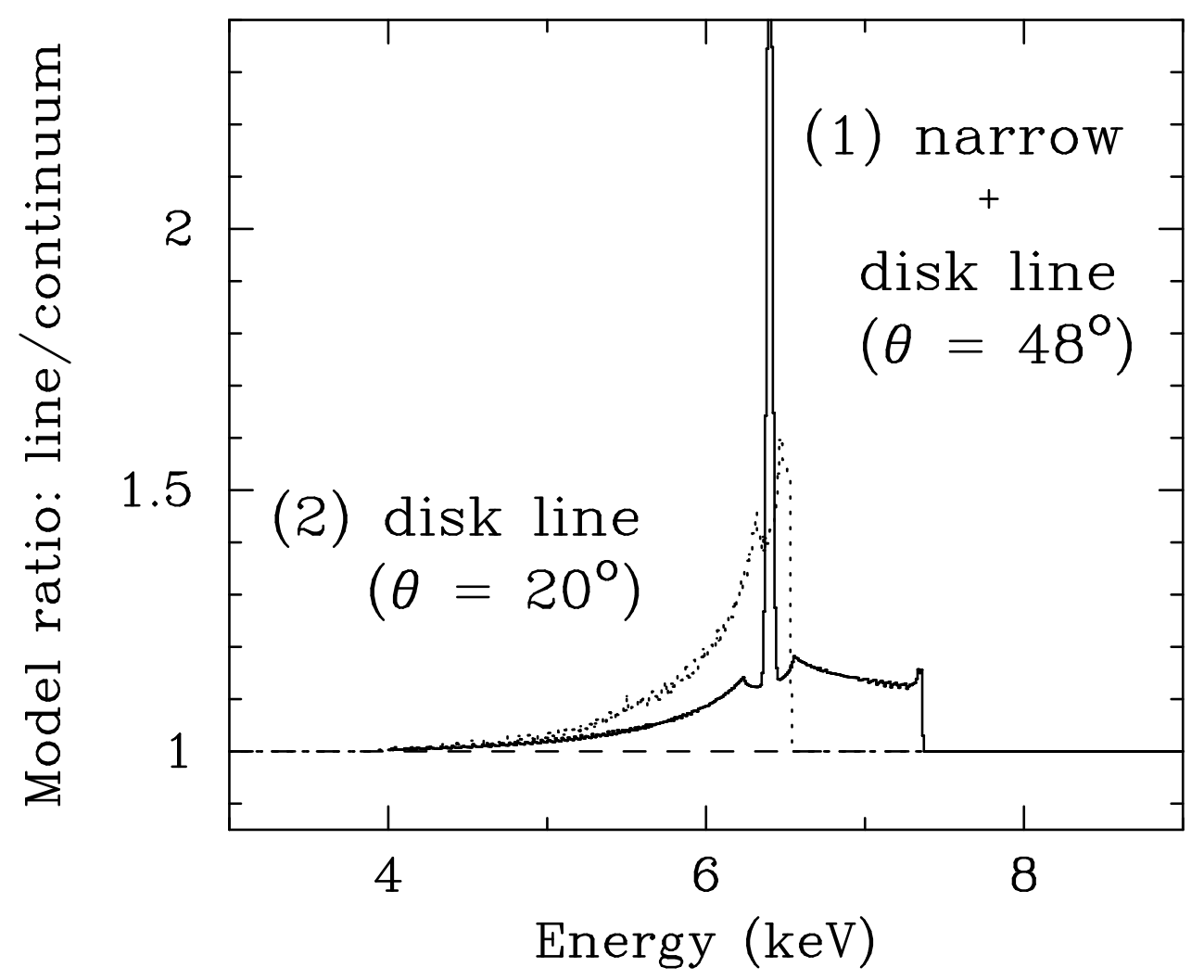

Fig. 3.- Theoretical line profiles for (1) an unresolved line superimposed on a line from an accretion disk viewed at $48^{\circ}$ (solid line) and (2) a line from an accretion disk viewed at an inclination of $20^{\circ}$ (T98, dotted line). This figure was produced by calculating the model spectrum (line plus power-law continuum) and dividing by the continuum model. 